\title{
Use of liquid biopsies to monitor disease progression in a sarcoma patient: a case report
}

Heidi M. Namløs ${ }^{1 *}$, Olga Zaikova², Bodil Bjerkehagen³ ${ }^{3}$ Daniel Vodák, Eivind Hovig ${ }^{1,4,5}$, Ola Myklebost ${ }^{1,5}$, Kjetil Boye ${ }^{1,6}$ and Leonardo A. Meza-Zepeda ${ }^{1,5,7^{*}}$

\begin{abstract}
Background: Many patients experience local recurrence or metastases after receiving potentially curative treatment, and early detection of these events is important for disease control. Recent technological advances make it possible to use blood plasma containing circulating cell-free tumour DNA (ctDNA) as a liquid biopsy. In this case report we show how serial liquid biopsies can be used to monitor the disease course and detect disease recurrence in a sarcoma patient.

Case presentation: A 55-year-old male presented with a rapidly growing, painful palpable mass in the left groin region, and a biopsy revealed a high-grade malignant spindle cell sarcoma. No metastases were detected on radiologic imaging scans. Using targeted resequencing with a custom 900 cancer gene panel, eight somatic mutations among them KRAS and NF1, were identified in the primary tumour. Targeted resequencing of plasma cell-free DNA (ctDNA) collected before and after surgery and at disease progression confirmed the presence of six of eight mutations at all three time points. The ctDNA level, estimated from the somatic allele frequencies of these six mutations, was high in plasma taken at the time of surgery, at levels similar to the primary tumour. Detection of low levels of ctDNA three days after surgery indicated persistent microscopic disease. Repeated radiologic imaging six weeks postoperatively showed widespread metastatic disease in the lungs, skeleton and the pelvic region. At this time point there was a dramatic increase in the ctDNA level, reflecting the disease progression of the patient. The patient had an unusually aggressive cancer, and succumbed to the disease 13 weeks after surgery.
\end{abstract}

Conclusions: This case report demonstrated that targeted resequencing of ctDNA from longitudinal collected plasma can be used to monitor disease progression in a soft tissue sarcoma patient, including manifestation of metastatic disease. The ctDNA represented the genomic profile of the tumour, supporting clinical use of liquid biopsies to identify tumour-specific mutations as well as recurrent disease.

Keywords: Circulating cell-free DNA, ctDNA, ThunderBolts, KRAS, NF1, Cancer, Case report

\section{Background}

Cancers arise through a sequential alteration of the genome, resulting in a heterogeneous tumour that continuously evolves as the disease progress. Material for diagnostic examination of solid tumours is routinely obtained through fine-needle biopsies. This procedure gives limited amount of material, and only provides a

\footnotetext{
* Correspondence: heidina@rr-research.no; Leonardo.Meza-Zepeda@rrresearch.no

'Department of Tumor Biology, Institute for Cancer Research, The Norwegian Radium Hospital, Oslo University Hospital, Oslo, Norway

Full list of author information is available at the end of the article
}

single snap-shot of the genetic alterations in a restricted part of the tumour. After treatment with curative intent, radiological imaging of patients to monitor local or distant recurrences is not done on a routine basis, and imaging methods also have limited sensitivity to detect micrometastases.

Recent advances make it possible to use blood plasma as a liquid biopsy, examining the circulating cell-free DNA (cfDNA) shed from both normal and tumour cells into peripheral blood [1-3]. The circulating cell-free tumour DNA (ctDNA) has been shown to contain the 
various tumour-specific alterations seen in the primary and metastatic tumours, and may more accurately represent the genetic profile of the whole tumour mass compared to DNA from a single biopsy of a heterogeneous lesion [4]. By repeated sampling of liquid biopsies, somatic mutations identified in cfDNA can be used as unique non-invasive tumourspecific biomarkers for monitoring tumour burden throughout the disease course. Similar procedures are now in use for screening of foetal genetic aberrations using the mothers blood, and in several cases aberrations from malignant tumours have been detected presymtomatic in pregnant women [5].

Several reports have demonstrated that highthroughput sequencing of cfDNA may be used for prognosis and molecular stratification, early detection of recurrence and metastasis, monitoring response to treatment and identification of resistance mechanisms [6]. Sequencing of cfDNA has been performed for cancers like colorectal, ovarian and breast, showing that the level of tumour-specific mutations reflects the course of the disease and the treatment response [7-9].

Sarcomas make up a heterogeneous group of malignant tumours of mainly mesenchymal origin. The overall five-year survival of all soft tissue sarcoma patients is approximately $70 \%[10,11]$, and about $75 \%$ of soft tissue sarcomas are highly malignant. Soft tissue sarcomas often recur locally and/or metastasize, and the median time to local recurrence is around $1-1 \frac{1}{2}$ year and to metastasis about 1 year $[12,13]$, both decreasing long-term survival. From a molecular genetics perspective, sarcomas are genetic diverse and may have numerous somatic mutations [14]. The use of high-throughput sequencing of cfDNAs longitudinally collected during disease progression, making simultaneous screening for multiple mutations during the disease course possible, has not yet been reported for sarcomas. However, a recent study described exome sequencing of a primary soft tissue sarcoma tumour and a single plasma sample collected at time of metastasis, and showed that new mutations had appeared in ctDNA after progression on chemotherapy and targeted treatment [15]. Monitoring of disease burden in one osteosarcoma patient has previously been demonstrated using PCR to detect somatic rearrangements in plasma [16], and allele-specific PCR has been used to obtain mutation profiles from plasma of GIST patients [17].

As part of an ongoing prospective study (CircSarc), we collected primary tumour and plasma samples taken before and after surgery and at disease progression from a soft tissue sarcoma patient. Targeted resequencing was used to identify somatic mutations in the primary tumour and monitor the level of ctDNA from plasma samples during the course of the disease.

\section{Case presentation}

A 55-year-old male presented with a rapidly growing, painful palpable mass in the left groin region involving the femoral nerve and blood vessels. Magnetic resonance imaging performed 18 days before surgery revealed a $10.5 \times 7.6 \times 11.0 \mathrm{~cm}$ large intramuscular tumour. No metastases were detected on CT scans of the chest, abdomen and pelvic area performed 14 days before surgery. Microscopic evaluation of a biopsy revealed a highgrade malignant spindle cell sarcoma (Fig. 1). Due to extensive locoregional growth into the skeleton and intractable pain, a hemipelvectomy was performed. Small focus with metastatic disease was detected in two lymph nodes removed during the surgery. Macroscopic examination showed a well demarcated nodular tumour with white and fleshy cutting surface with small necrotic areas and bleeding. Immunohistochemical analysis showed positive finding for CD99 and AE1/AE3, and negative staining for S-100, SMA, EMA and CD31. Cytogenetic analysis showed massive clonal chromosomal rearrangements, and PCR and FISH were negative for fusion genes normally seen in synovial sarcoma. The differential diagnoses were synovial sarcoma and malignant peripheral nerve sheath tumour. Lymph node metastasis is more commonly seen in synovial sarcoma and the immunohistochemical finding is also in favour of a synovial sarcoma, but the genetic findings did not support that diagnosis. According to the WHO classification [18], the tumour was classified as an undifferentiated spindle cell sarcoma.

Targeted resequencing of the tumour and normal genomic DNA was performed following a SureSelect protocol (Agilent Technologies, Santa Clara, California, US) and a custom in-solution capture panel, containing exons of 900 cancer-related genes, developed by the Norwegian Cancer Genomics Consortium (NCGC, cancergenomics.no) [19]. The methods are provided in (Additional file 1). The sequencing revealed eight somatic mutations in the primary tumour. Among these, seven point mutations were identified in the genes COL2A1 (intronic), NF1 (p.K354R), PTGS2 (intronic), LRP2 (p.Q4132E), KRAS (p.G12V), PRRC2C (p.R1257G) and GATA6 (p.A29A), as well as a frameshift deletion in PRG4 (p.R791fs) (Table 1 and Additional file 2). Copy number analysis revealed a homozygote deletion of TP53 (Additional file 3). Targeted resequencing using a smaller ThunderBolts Cancer panel (Raindance Technologies, Billerica, Massachusetts, US) confirmed the identified KRAS mutation in the primary tumour at an allele frequency of $66 \%$, similar to the $60 \%$ frequency found using the 900 gene panel (Additional file 2). The additional seven mutated genes were not included within the ThunderBolts Cancer panel. 

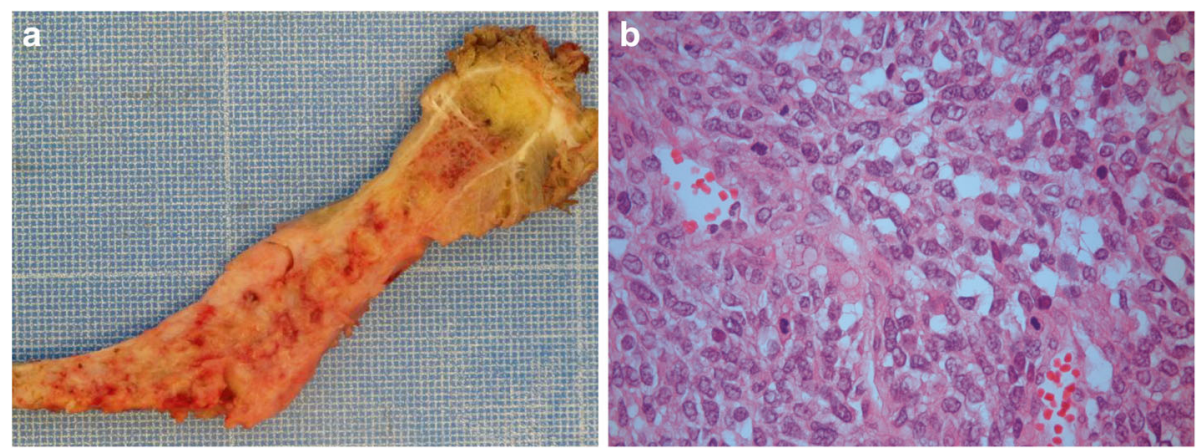

Fig. 1 Examination of tumour tissue (a). Macroscopic examination of tumour in femur after hemipelvectomy. b Histological picture of heamatoxylin and eosin stained slide of patient biopsy material classified as undifferentiated spindle cell sarcoma

The patient was scheduled for adjuvant chemotherapy, but repeated radiologic imaging six weeks postoperatively showed widespread macroscopic metastatic disease in the lungs and skeleton, as well as numerous soft tissue metastases in the pelvic region. Targeted resequencing of the plasma samples, using the NCGC 900 cancer gene panel, confirmed the presence of six of the eight above mutations in all three plasma samples with allele frequencies ranging from 2.1-75\% (Fig. 2 and Additional file 2). The mutation levels in plasma before surgery were comparable to the ones in the primary tumour with a Pearson's correlation of 0.93 between frequencies in tumour and plasma. The mutations in GATA6 and $P R G 4$, with the lowest allele frequencies in the primary tumour, were not detected in the plasma samples. The mean sequencing depth of the six detected mutations in all three plasma samples was 260x (11-474x), and the two undetected had a depth of 360x (199-433x). One new mutation in the splice site of $R A D 52$, a $\mathrm{C}>\mathrm{T}$ mutation with an allele frequency of $5.5 \%$, was detected in the cfDNA collected before surgery. This region was sequenced at high coverage, but the mutation was not observed in the primary tumour nor in the plasma samples collected at later time points (Additional file 2).
Targeted resequencing using the ThunderBolts Cancer panel confirmed the presence of KRAS (p.G12V)) in all plasma samples (Additional file 2). No other mutations with $>5 \%$ allele frequency were detected in more than one sample.

The level of total cfDNA was monitored during disease progression. High quantity of cfDNA was detected one day before surgery (110 $\mathrm{ng} / \mathrm{ml}$ plasma), and a decrease was seen three days after surgery $(76 \mathrm{ng} / \mathrm{ml}$ plasma). Six weeks after surgery, the quantity of cfDNA had increased to more than twice the initial level present before the surgery (316 $\mathrm{ng} / \mathrm{ml}$ plasma) (Fig. 2).

The ctDNA level was estimated from the somatic allele frequency of the recurrent mutations in the genes COL2A1, NF1, PTGS2, LRP2, KRAS and PRRC2C. The ctDNA level in plasma collected one day before the surgery (Plasma1) was high, and comparable to the level in primary tumour. Three days after surgery, the ctDNA level had dropped, but was still detectable in plasma (Plasma2). In the sample collected six weeks after surgery (Plasma3), there was again an increase in ctDNA level similar to the levels before surgery (Fig. 2). When also taking into account the amount of cfDNA released, the number of mutated genomes per $\mathrm{ml}$ of plasma were

Table 1 Overview of somatic mutations in primary tumour. The sequencing libraries were generated using the SureSelect ${ }^{\mathrm{XT}}$ protocol and a 900 cancer-related custom SureSelect in-solution capture gene panel

\begin{tabular}{llllllll}
\hline Gene & Position & Reference allele & $\begin{array}{l}\text { Mutated } \\
\text { allele }\end{array}$ & Codon change & $\begin{array}{l}\text { Allele frequency } \\
\text { tumour }\end{array}$ & $\begin{array}{l}\text { Depth } \\
\text { tumour }\end{array}$ & Consequence \\
\hline COL2A1 & chr12:48372367 & G & C & NA & 96.0 & 725 & Intron variant \\
NF1 & chr17:29527612 & A & G & p.K354R & 92.9 & 438 & Missense variant \\
PTGS2 & chr1:186644059 & C & A & NA & 64.0 & 39 & Intron variant \\
LRP2 & chr2:170009376 G & C & p.Q4132E & 61.0 & 443 & Missense variant \\
KRAS & chr12:25398284 C & A & p.G12V & 60.4 & 699 & Missense variant \\
PRRC2C & chr1:171510380 & A & G & p.R1257G & 29.0 & 807 & Missense variant \\
GATA6 & chr18:19751192 & $G$ & T & p.A29A & 18.9 & 920 & Synonymous variant \\
PRG4 & chr1:186277624 & CGTACTACACCT & C & p.R791fs,p.R884fs,p.R832fs,p.R925fs & 10.6 & 810 & Frameshift variant \& \\
& & & & & & & feature truncation \\
\hline
\end{tabular}




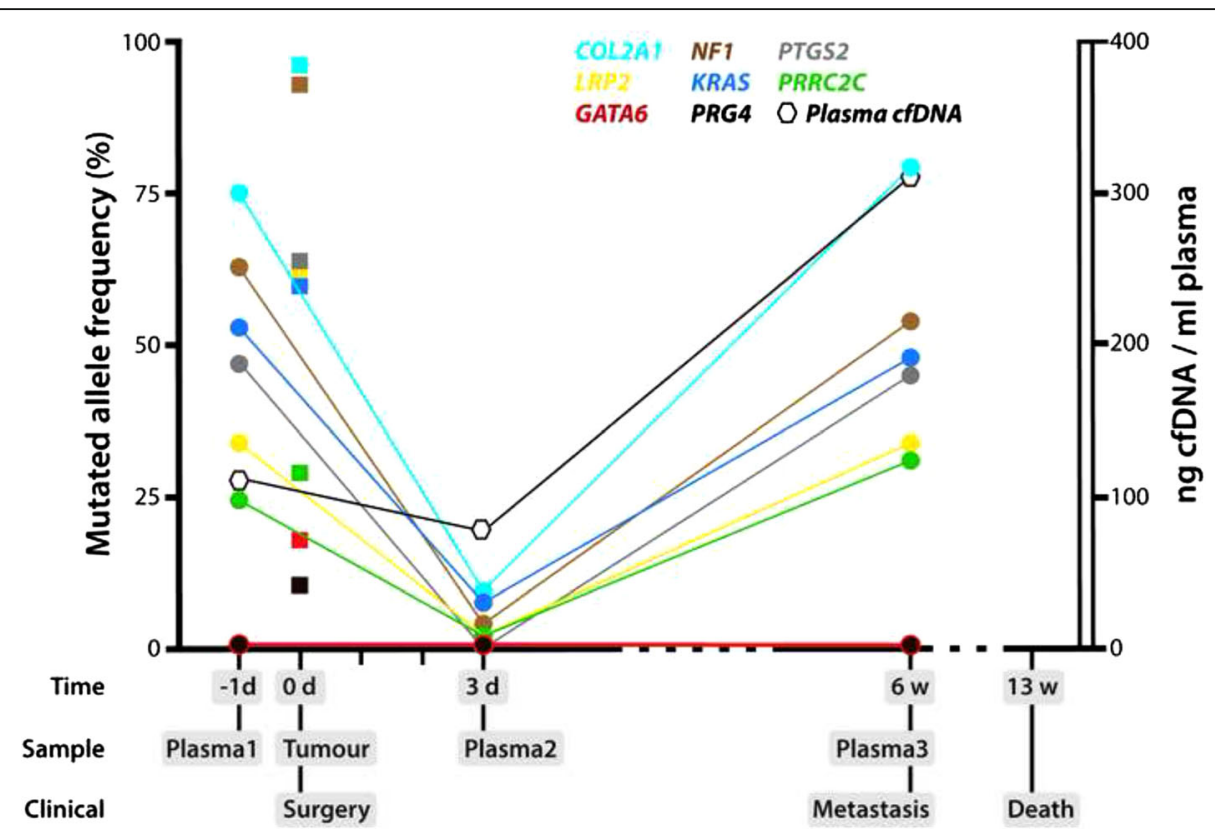

Fig. 2 Levels of mutations in ctDNA and total cfDNA level in serial samples during disease progression. The allele frequency of mutations in COL2A1, NF1, PTGS2, LRP2, KRAS and PRRC2C was quantified in primary tumour (squares) at time of surgery (0d) and in serial plasma samples (circles) collected one day $(-1 \mathrm{~d})$ before surgery, three days (3d) and six weeks $(6 \mathrm{~W})$ after surgery using targeted resequencing. The total level of cfDNA (normal and tumour) was quantified in plasma samples (hexagon) using Qubit fluorometer. Distal metastases were identified after six weeks $(6 \mathrm{w})$, and the patient succumbed to the disease after 13 weeks (13w) with aggressive metastatic disease

three times higher at this time point than before surgery (Additional file 4). This reflected the disease progression of the patient and correlated with the tumour burden, as multiple distant metastases were detected at this time. The patient's general condition was considered too poor for administering chemotherapy, and he succumbed to the disease 13 weeks after surgery.

\section{Discussions}

In this study, we prospectively collected primary tumour and normal sample material at surgery and several plasma samples during the disease course of a highgrade soft tissue sarcoma patient. Targeted resequencing of the primary tumour and the normal sample identified eight somatic mutations of which six were also present in the plasma samples. Among the mutations, KRAS (p.G12V) and NF1 (p.K354R) were predicted by dbNSFP [20] to have a deleterious effect on the protein function. It has been reported that simultaneous inactivation of TP53 and activation of KRAS induced quick formation of spindle-cell sarcoma in soft tissues in double transgenic mice [21]. The homozygous deletion of TP53 found in the primary tumour strengthens the histology observed in the primary tumour. The patient in our study had an unusually aggressive spindle-cell sarcoma, supporting $K R A S$ not only as biomarker, but as a driving gene of the disease progression. NF1, a tumour suppressor that functions as a negative regulator of the Ras pathway, is among the most frequently mutated genes in several subtypes of sarcomas. Germline and somatic loss of NF1 in neurofibromatosis patients cause malignant peripheral nerve sheath tumours [22] and GISTs [23]. In addition, somatic NF1 mutations, including deletions, have been reported in a wide variety of paediatric and adult soft-tissue sarcomas with complex karyotypes [24, 25]. Although no therapeutics that target KRAS or NF1 are available, our study shows that repeated sampling using liquid biopsies opens new possibilities to identify and monitor biomarkers that can be used in targeted therapies.

The synonymous mutation in GATA6 and the frameshift deletion in PRG4 could not be detected in the plasma samples. Although these regions had a high coverage, these mutations had the lowest allele frequency in the primary tumour and this may thus be a sensitivity issue. However, it is also possible that the cells containing these mutations did not release DNA into circulation. This shows that the ctDNA gives a good representation of the genomic profile of the tumour, but also emphasize the need to use several mutations when monitoring disease development. A mutation in RAD52, a gene involved in DNA recombination, was detected in the plasma collected before surgery, but was not observed in the primary tumour nor in the plasma sample collected after surgery or at recurrence. One explanation for this may be tumour heterogeneity where the cells 
containing this mutation were not present in the part of the primary tumour that was sequenced and was not retained during disease progression. Discrepancy of mutation pattern between DNA from primary tumours and plasma have also previously been reported [26, 27], showing that liquid biopsies may better capture all mutations present in the primary tumour and/or liver metastasis and can thus be used to overcome the challenges posed by intra-tumour genetic heterogeneity [4]. We show that ctDNA from plasma represents an attractive and easily available source of sarcoma tumour DNA, although it remains to be seen how ctDNA levels vary across patients and stages.

The allele frequencies of the six mutated genes in the cfDNA represent the ctDNA level during disease progression. Three days after surgery, ctDNA was still detectable in the liquid biospy. cfDNA has a rapid clearance, with reported half-life from $15 \mathrm{~min}$ [28] to $13 \mathrm{~h}$ for foetal cfDNA in plasma [29]. Although not detectable by CT before surgery, metastatic disease was detected in two lymph nodes removed during the hemipelvectomy, and a small amount of ctDNA detected was likely released from additional undiscovered local metastases that could not be detected by conventional diagnostic modalities. The patient had a very aggressive course of the disease, and metastases were detected both in soft tissue, skeleton and lungs only a few weeks after surgery. The plasma collected six weeks after surgery showed an increase in ctDNA relative to the levels before surgery, reflecting the presence of a tumour and rapid disease progression.

The cfDNA can originate from both normal and tumour cells. Based on the high mutated allele frequencies determined in plasma, the initial level of cfDNA is dominated by DNA from the tumour. Most of the cfDNA present three days after surgery is believed to originate from tissue injury and inflammation of normal cells as a consequence of the extensive surgery, which would explain the apparently higher normal contribution to the cfDNA at this time point. After six weeks, there was a large increase in cfDNA accompanied with an increase of the mutated allele frequencies. Thus, the quantities of cfDNA present in the plasma reflected the clinical status of the patient due to the fact that most of the cfDNA released during disease progression was tumour derived.

\section{Conclusions}

This study is the first report of using targeted resequencing of cfDNA from serial plasma samples to monitor disease progression in a soft tissue sarcoma patient. The findings show that the level of tumour-specific mutations in liquid biopsies is correlated to disease course in sarcomas, including clinical manifestation of metastatic disease. The longitudinally collected ctDNA allow for near real-time monitoring of the tumour genome during disease progression, and the ctDNA gives a good representation of the genomic profile of the tumour supporting the use of ctDNA from plasma as a liquid biopsy.

\section{Additional files}

Additional file 1 Somatic mutations identified in primary tumour and plasma samples using NCGC 900 and Thunderbolt Cancer Panel. (DOCX 18 kb)

Additional file $\mathbf{2}$ Somatic mutations identified in primary tumour and plasma samples using NCGC 900 and Thunderbolt Cancer Panel. (XLS 62 kb)

Additional file 3 Plot showing copy number of chromosome 17p, revealing homozygote deletion of the TP53 gene. (TIF $1032 \mathrm{~kb}$ )

Additional file 4 Plot showing mutated genomes per $\mathrm{ml}$ of plasma, for the mutated genes, in serial plasma samples. (TIFF $283 \mathrm{~kb}$ )

\section{Abbreviations}

BWA: Burrows-Wheeler alignment; cfDNA: Circulating cell-free DNA: COL2A1: Collagen, type II, alpha 1; ctDNA: Circulating cell-free tumour DNA; GATA6: GATA binding protein 6; GATK: The genome analysis toolkit; KRAS: Kirsten rat sarcoma viral oncogene homolog; LRP2: Low density lipoprotein receptor-related protein 2; NCGC: Norwegian cancer genomics consortium; NF1: Neurofibromin 1; PRG4: Proteoglycan 4; PRRC2C: Proline-rich coiled-coil 2C; PTGS2: Prostaglandin-endoperoxide synthase 2; RAD52: RAD52 homolog (S. Cerevisiae); TP53: Tumour protein P53

\section{Acknowledgements}

We thank Synnøve Granlien in her role as study nurse, Ole-Jacob Norum for clinical contribution, Kirsten Sundby Hall for involvement on clinical design, Ellen Tenstad and Stine $\mathrm{H}$. Kresse for contributions during manuscript preparation and the personnel at the Genomics Core Facility at Oslo University Hospital (oslo.genomics.no) for technical assistance with the sequencing.

\section{Funding}

Norwegian Cancer Society, grant number PR-2007-0163.

Norwegian Research Council, grant number 221580.

Availability of data and materials

Any additional supporting data involving details of clinical and genetic analysis can be found in the medical record system at Oslo University Hospital, and may be available upon request.

\section{Authors' contributions}

HMN designed the case report, performed the experiments and drafted the manuscript. OZ participated in the design and drafting of the manuscript and collected the informed consent. BB performed the pathological examination and participated in the drafting of the manuscript. DV and EH performed the bioinformatics analysis. OM participated in the design of the case report. KB participated in the design and conceived of the study. LAMZ designed the case study and participated in the drafting of the manuscript. All authors read and approved the final manuscript.

\section{Competing interests}

The authors declare that they have no competing interests. No writing assistance was utilized in the production of this manuscript.

\section{Consent for publication}

Written informed consent to publish the data was obtained from the patient.

\section{Ethics approval and consent to participate}

Current investigation was performed within the frame of the prospective study "CircSarc - development of personalized biomarkers for disease monitoring in sarcomas". The project (S-06133) was approved by the Regional Ethical Committee for Southern Norway. Written informed consent to participate in the study was obtained from the patient. 


\section{Author details}

'Department of Tumor Biology, Institute for Cancer Research, The Norwegian Radium Hospital, Oslo University Hospital, Oslo, Norway. ${ }^{2}$ Department of Surgery, Oslo University Hospital, Oslo, Norway. ${ }^{3}$ Department of Pathology, Oslo University Hospital, Oslo, Norway. ${ }^{4}$ Institute of Cancer Genetics and Informatics, The Norwegian Radium Hospital, Oslo University Hospital, Oslo, Norway. ${ }^{5}$ Norwegian Cancer Genomics Consortium (CancerGenomics.no), Oslo, Norway. ${ }^{6}$ Department of Oncology, Oslo University Hospital, Oslo, Norway. ${ }^{7}$ Genomics Core Facility, Department of Core Facilities, Institute for Cancer Research, The Norwegian Radium Hospital, Oslo University Hospital, Oslo, Norway.

Received: 25 November 2015 Accepted: 13 December 2016 Published online: 06 January 2017

\section{References}

1. Bettegowda C, Sausen M, Leary RJ, Kinde I, Wang Y, Agrawal N, Bartlett BR, Wang $\mathrm{H}$, Luber $\mathrm{B}$, Alani RM, et al. Detection of circulating tumor DNA in earlyand late-stage human malignancies. Sci Transl Med. 2014;6(224):224ra224.

2. Thierry AR, Mouliere F, El Messaoudi S, Mollevi C, Lopez-Crapez E, Rolet F, Gillet B, Gongora C, Dechelotte P, Robert B, et al. Clinical validation of the detection of KRAS and BRAF mutations from circulating tumor DNA. Nat Med. 2014;20(4):430-5.

3. Leary RJ, Kinde I, Diehl F, Schmidt K, Clouser C, Duncan C, Antipova A, Lee C, McKernan K, De La Vega FM, et al. Development of personalized tumor biomarkers using massively parallel sequencing. Sci Transl Med. 2010;2(20):20ra14.

4. De Mattos-Arruda L, Weigelt B, Cortes J, Won HH, Ng CKY, Nuciforo P, Bidard F-C, Aura C, Saura C, Peg V, et al. Capturing intra-tumor genetic heterogeneity by de novo mutation profiling of circulating cell-free tumor DNA: a proof-of-principle. Ann Oncol. 2014;25(9):1729-35.

5. Amant F, Verheecke M, Wlodarska I, Dehaspe L, Brady P, Brison N, Van Den Bogaert K, Dierickx D, Vandecaveye $V$, Tousseyn $T$ et al. Presymptomatic identification of cancers in pregnant women during noninvasive prenatal testing. JAMA Oncol. 2015, doi:10.1001/jamaoncol.2015.1883.

6. Crowley E, Di Nicolantonio F, Loupakis F, Bardelli A. Liquid biopsy: monitoring cancer-genetics in the blood. Nat Rev. 2013;10(8):472-84.

7. Forshew T, Murtaza M, Parkinson C, Gale D, Tsui DWY, Kaper F, Dawson S-J, Piskorz AM, Jimenez-Linan M, Bentley D, et al. Noninvasive identification and monitoring of cancer mutations by targeted deep sequencing of plasma DNA. Sci Transl Med. 2012;4(136):136ra168.

8. Diehl F, Schmidt K, Choti MA, Romans K, Goodman S, Li M, Thornton K, Agrawal N, Sokoll L, Szabo SA, et al. Circulating mutant DNA to assess tumor dynamics. Nat Med. 2008;14(9):985-90.

9. Siravegna G, Mussolin B, Buscarino M, Corti G, Cassingena A, Crisafulli G, Ponzetti A, Cremolini C, Amatu A, Lauricella C, et al. Clonal evolution and resistance to EGFR blockade in the blood of colorectal cancer patients. Nat Med. 2015;21(7):795-801

10. Bauer HC, Trovik CS, Alvegard TA, Berlin O, Erlanson M, Gustafson P, Klepp R, Moller TR, Rydholm A, Saeter $G$, et al. Monitoring referral and treatment in soft tissue sarcoma: study based on 1851 patients from the Scandinavian Sarcoma Group Register. Acta Orthop Scand. 2001;72(2):150-9.

11. Jemal A, Siegel R, Ward E, Murray T, Xu J, Smigal C, Thun MJ. Cancer statistics, 2006. CA Cancer J Clin. 2006;56(2):106-30.

12. Sawamura $C$, Matsumoto $S$, Shimoji T, Tanizawa $T$, Ae K. What are risk factors for local recurrence of deep high-grade soft-tissue sarcomas? Clin Orthop Relat Res. 2012;470(3):700-5.

13. Sawamura C, Matsumoto S, Shimoji T, Okawa A, Ae K. How long should we follow patients with soft tissue sarcomas? Clin Orthop Relat Res. 2014;472(3):842-8.

14. Taylor BS, Barretina J, Maki RG, Antonescu CR, Singer S, Ladanyi M. Advances in sarcoma genomics and new therapeutic targets. Nat Rev Cancer. 2011; 11(8):541-57.

15. Butler TM, Johnson-Camacho K, Peto M, Wang NJ, Macey TA, Korkola JE, Koppie TM, Corless CL, Gray JW, Spellman PT. Exome sequencing of cell-free DNA from metastatic cancer patients identifies clinically actionable mutations distinct from primary disease. PLoS One. 2015:10(8):e0136407.

16. McBride DJ, Orpana AK, Sotiriou C, Joensuu H, Stephens PJ, Mudie L, Hämäläinen E, Stebbings LA, Andersson LC, Flanagan AM, et al. Use of cancer-specific genomic rearrangements to quantify disease burden in plasma from patients with solid tumors. Genes Chromosomes Cancer. 2010; 49(11):1062-9.
17. Maier J, Lange T, Kerle I, Specht K, Bruegel M, Wickenhauser C, Jost P, Niederwieser D, Peschel C, Duyster J, et al. Detection of Mutant Free Circulating Tumor DNA in the Plasma of Patients with Gastrointestinal Stromal Tumor Harboring Activating Mutations of CKIT or PDGFRA. Clin Cancer Res. 2013;19(17):4854-67.

18. Fletcher CDM, Hogendoorn PCW, Mertens F, Bridge J. WHO Classification of Tumours of Soft Tissue and Bone. Lyon, France: IARC Press; 2013.

19. Chilamakuri CSR, Lorenz S, Madoui M-A, Vodák D, Sun J, Hovig E, Myklebost O, Meza-Zepeda LA. Performance comparison of four exome capture systems for deep sequencing. BMC Genomics. 2014;15(1):449.

20. Liu X, Jian X, Boerwinkle E. dbNSFP v2.0: A Database of human nonsynonymous SNVs and their functional predictions and annotations. Hum Mutat. 2013;34(9):E2393-402.

21. Yang X, La Rosa FG, Genova EE, Huber K, Schaack J, Degregori J, Serkova NJ, Li Y, Su LJ, Kessler E, et al. Simultaneous activation of Kras and inactivation of p53 induces soft tissue sarcoma and bladder urothelial hyperplasia. PLoS One. 2013;8(9):e74809.

22. King AA, Debaun MR, Riccardi VM, Gutmann DH. Malignant peripheral nerve sheath tumors in neurofibromatosis 1. Am J Med Genet. 2000;93(5):388-92.

23. Maertens $\mathrm{O}$, Prenen $H$, Debiec-Rychter M, Wozniak A, Sciot R, Pauwels $P$, De Wever I, Vermeesch JR, de Raedt T, De Paepe A, et al. Molecular pathogenesis of multiple gastrointestinal stromal tumors in NF1 patients. Hum Mol Genet. 2006;15(6):1015-23.

24. Barretina J, Taylor BS, Banerii S, Ramos AH, Lagos-Quintana M, DeCarolis PL, Shah K, Socci ND, Weir BA, Ho A, et al. Subtype-specific genomic alterations define new targets for soft tissue sarcoma therapy. Nat Genet. 2010:42(8):715-21.

25. Paulson V, Chandler G, Rakheja D, Galindo RL, Wilson K, Amatruda JF, Cameron S. High-resolution array CGH identifies common mechanisms that drive embryonal rhabdomyosarcoma pathogenesis. Genes Chromosomes Cancer. 2011;50(6):397-408.

26. Rothé $F$, Laes J-F, Lambrechts $D$, Smeets D, Vincent $D$, Maetens $M$, Fumagall D, Michiels S, Drisis S, Moerman C, et al. Plasma circulating tumor DNA as an alternative to metastatic biopsies for mutational analysis in breast cancer. Ann Oncol. 2014;25(10):1959-65.

27. Murtaza M, Dawson S-J, Pogrebniak K, Rueda OM, Provenzano E, Grant J, Chin S-F, Tsui DWY, Marass F, Gale D, et al. Multifocal clonal evolution characterized using circulating tumour DNA in a case of metastatic breast cancer. Nat Commun. 2015;6:8760.

28. Lo YM, Zhang J, Leung TN, Lau TK, Chang AM, Hjelm NM. Rapid clearance of fetal DNA from maternal plasma. Am J Hum Genet. 1999;64(1):218-24.

29. Yu SCY, Lee SWY, Jiang P, Leung TY, Chan KCA, Chiu RWK, Lo YMD. Highresolution profiling of fetal DNA clearance from maternal plasma by massively parallel sequencing. Clin Chem. 2013;59(8):1228-37.

\section{Submit your next manuscript to BioMed Central and we will help you at every step:}

- We accept pre-submission inquiries

- Our selector tool helps you to find the most relevant journal

- We provide round the clock customer support

- Convenient online submission

- Thorough peer review

- Inclusion in PubMed and all major indexing services

- Maximum visibility for your research

Submit your manuscript at www.biomedcentral.com/submit
) Biomed Central 